\title{
Is less more? A preliminary investigation of the number of response categories in self-reported pain
}

This article was published in the following Dove Press journal:

Patient Related Outcome Measures

18 May 2010

Number of times this article has been viewed

\author{
Karon F Cook' \\ David Cella ${ }^{2}$ \\ Erin L Boespflug' \\ Dagmar Amtmann' \\ 'Department of Rehabilitation \\ Medicine, University of Washington, \\ Seattle, WA; ${ }^{2}$ Department of Medical \\ Social Sciences, Northwestern \\ University, Feinberg School of \\ Medicine, Chicago, IL, USA
}

\begin{abstract}
The purpose of this study was to conduct a preliminary investigation of the number of response options for self-reports of pain interference. Responses to interference items of the 11-category Brief Pain Inventory (BPI) were obtained in a sample of 434 persons from two sites and modeled using the partial credit model. In successive calibrations, response categories were collapsed and new scores were generated. Scores based on two to three categories produced poor results. Four to five categories yielded better results. However, scoring using more than five categories did not appreciably improve the reliability, person separation, or validity of scores. These results suggest that fewer response categories - as few as five or sixmay function as well as the 11 response categories that are conventionally used. The results are preliminary since the number of response categories actually presented was not manipulated in the study design. Future research should compare the reliability and validity of scores based on the BPI interference items when items are presented with the conventionally 11-response format, versus presentation with fewer response options.
\end{abstract}

Keywords: psychometrics, outcomes, quality of life, measurement, pain

\section{Background}

Pain is defined as a subjective and unpleasant sensory/emotional experience that is associated with actual or potential tissue damage. ${ }^{1,2}$ The measurement of pain presents difficult challenges, and issues regarding the scaling of pain have yet to be thoroughly conceptualized. In addition to informing pain measurement, psychometric evaluations of self-reported pain instruments potentially contribute substantively to understanding how pain is perceived.

Clinical tradition has established a 'standard' zero-to-ten rating scale for pain and related functional problems. A numerical rating scale with 11 response categories was recommended by IMMPACT ${ }^{3}$ and has become a gold standard in research applications. In their recommendations, the IMMPACT group did not directly address the number of response categories and did not reference any studies to support the choice of 11 categories. The common use of 11 or more response categories for the measurement of symptoms suggests an assumption that 'more is better.'

Much of the research on the impact of the number of response categories was conducted two or more decades ago and published outside the field of health outcomes, mostly in psychology and marketing research. Taken together, a manuscript by Preston and Colman ${ }^{4}$ and another by Weng ${ }^{5}$ provide a thorough review of this literature. To summarize, Symonds ${ }^{6}$ argued in 1924 for the use of more than 20 response categories, but research since then has not supported the use of this many. Bending 7,8
Correspondence: Karon F Cook 801 Cortlandt St., Houston, TX 77007, USA $\mathrm{Tel}+17132913918$

Email karonc2@u.washington.edu 
found similar reliability with two- to nine-category scales, but decreased reliability with 11-category scales. Using a simulation study, Cicchetti ${ }^{9}$ and colleagues observed increases in reliability from two- to seven-point scales, but concluded that seven response categories were 'at least functionally interchangeable with as many as 100 such ordered categories' (p. 35). Consistent with these results are the conclusions of others who argue that seven is the optimal number of response categories for maximizing reliability. ${ }^{10-12}$ Others have indicated a preference for fewer than seven categories. ${ }^{13-16}$

Though including a large number of categories may not add substantially to the reliability of scores, too few can be detrimental. A study by Preston and Colman ${ }^{4,5}$ and another by Weng ${ }^{4,5}$ suggested that adequate reliability is not achieved with less than three or four response categories. Reliability, validity and discrimination improved as more response categories were added, up to about seven categories. ${ }^{4,5}$

One possible explanation for the psychometric results obtained in the studies described above is that there are substantial limits in how many levels of a trait persons are able to discriminate. In 1956, George Miller published an influential article titled, 'The Magical Number Seven, Plus or Minus Two: Some Limits on Our Capacity for Processing Information' ${ }^{17}$ In it, he summarized several psychological experiments that evaluated the number of levels of a stimulus persons could discriminate (referred to as 'channel capacity'). With few exceptions, the upper limit of this discrimination was 'seven, plus or minus two' levels.

The purpose of the current study was to use a Rasch measurement model to conduct a preliminary investigation into the use of different numbers of response options for scoring items of the interference subscale of the Brief Pain Inventory (BPI). Rasch models enable evaluations of scale structure at both the item and the response category level. The BPI interference scale is a seven-item measure of pain interference that employs an 11-point scale ranging from 0 ('Does not interfere') to 10 ('Completely interferes'). ${ }^{18} \mathrm{We}$ compared the results of scoring the BPI using all 11 categories with results obtained by collapsing adjacent categories and scoring items with two to 10 response categories.

\section{Methods}

\section{Brief Pain Inventory}

The BPI is a multidimensional instrument that includes items evaluating pain location, intensity, quality, and interference. ${ }^{18}$ It has been translated into many languages including Korean, ${ }^{19}$ Spanish $^{20}$ and Norwegian, ${ }^{21}$ and its psychometric properties have been evaluated extensively in many clinical populations. ${ }^{19,21-32}$

\section{Data}

The data used in this study were archival. Responses $(\mathrm{N}=434)$ to the BPI Interference items were collected in two geographical locations and several clinical populations. Data from several unrelated studies were combined for the dataset that was used. Persons with cancer were recruited through Evanston Northwestern Healthcare Center on Outcomes Research and Education (ENH-CORE) in Chicago, Illinois $(\mathrm{N}=202)$. Persons with multiple sclerosis and amputation were recruited through the University of Washington Center on Outcomes Research in Rehabilitation (UW-CORR) in Seattle, Washington $(\mathrm{N}=232)$. In addition to the BPI responses, information was collected on demographic and clinical variables.

\section{Item calibrations}

Using WINSTEPS software, ${ }^{33}$ item responses were modeled to the partial credit model (PCM), ${ }^{34}$ a Rasch model appropriate for calibrating items that have more than two response categories. With the PCM Masters conceptualized the category responses associated with a given polytomous item as a series of successive 'steps.' An examinee either succeeds or fails each step within an item. The total number of steps passed serves as an examinee's category score. Masters defined the probability of a given category score as:

$$
P_{i x}(\theta)=\frac{\exp \left[\sum_{k=0}^{x}\left(\theta b_{i k}\right)\right]}{\sum_{h=0}^{m_{i}} \exp \left[\sum_{k=0}^{h}\left(\theta b_{i k}\right)\right]}\left(x=0, \ldots m_{i}\right)
$$

where

$b_{i x}=$ the difficulty of the step associated with the category score, $x$, for item $i$, and,

$m_{i}=$ the highest possible score on item $i$.

Though the response categories must be ordered when using the PCM, the step difficulties do not have to be ordered; ie, reversals are allowed.

\section{Datasets}

Using the original data, a total of 11 datasets were created, and calibrated to the PCM. One of these was the dataset with the original $0-10$ coding. For an additional nine datasets, adjacent categories were collapsed uniformly across items in order to obtain datasets with 2-10 categories per 
item. For example, for the 10-response category dataset, the last two response categories (' 9 ' and ' 10 ') were collapsed so that persons endorsing either ' 9 ' or ' 10 ' were assigned the same category score. For the sake of clarity, we refer to the nine datasets developed to have an equal number of categories across all items as the 'Uniform Response' datasets.

Table 1 describes how responses in each dataset were recoded. For the Uniform Response datasets, we favored collapsing those adjacent categories which, across items, had the least responses. Because the scores were skewed positively, we more often collapsed adjacent higher response categories. Because the items within a given uniform response dataset all had the same number of response categories, we attempted to make decisions regarding which categories to collapse based on the characteristics of all items within a dataset. However, except for favoring the collapsing of sparsely-populated response categories, decisions regarding which categories to collapse were somewhat arbitrary, and a large number of other combinations of recodings would have been equally defensible.

In a final dataset, decisions about which pairs of response categories to collapse were made at the item level, and the number of recoded categories was not the same for all items. We refer to this as the 'Variable Response' dataset. As a first step in developing this dataset, we collapsed categories so that there would be at least 20 observations in each category. The resulting data were then recalibrated using the $\mathrm{PCM}$, and the category characteristic functions (CCF) were inspected. CCFs are plots that describe the mathematical relationship between the level of the trait being measured and the probability of a given response. ${ }^{35,36}$ When all response categories are contributing substantially and distinctly to the measurement of the trait, every response category will be the

Table I Recoding of I I category Brief Pain Inventory responses

\begin{tabular}{lllllllllllr}
\hline $\begin{array}{l}\text { Number of } \\
\text { categories in } \\
\text { recoding }\end{array}$ & \multicolumn{10}{c}{ How response categories were recoded } \\
\hline II (original) & 0 & I & 2 & 3 & 4 & 5 & 6 & 7 & 8 & 9 & 10 \\
10 & 0 & I & 2 & 3 & 4 & 5 & 6 & 7 & 8 & 9 & 9 \\
9 & 0 & I & 2 & 3 & 4 & 5 & 6 & 7 & 7 & 8 & 8 \\
8 & 0 & I & 2 & 3 & 4 & 4 & 5 & 6 & 6 & 7 & 7 \\
7 & 0 & I & 2 & 3 & 3 & 4 & 4 & 5 & 5 & 6 & 6 \\
6 & 0 & I & 1 & 2 & 2 & 3 & 3 & 4 & 4 & 5 & 5 \\
5 & 0 & 1 & 1 & 2 & 2 & 3 & 3 & 3 & 4 & 4 & 4 \\
4 & 0 & 0 & 1 & 1 & 1 & 2 & 2 & 2 & 3 & 3 & 3 \\
3 & 0 & 0 & 0 & 1 & 1 & 1 & 1 & 2 & 2 & 2 & 2 \\
2 & 0 & 0 & 0 & 0 & 0 & 1 & 1 & 1 & 1 & 1 & I \\
\hline
\end{tabular}

Table 2 Coding of dataset developed by inspection of category characteristic functions

\begin{tabular}{lllllllllllr}
\hline \multicolumn{10}{c}{ How response categories were recoded } \\
\hline II (original) & 0 & I & 2 & 3 & 4 & 5 & 6 & 7 & 8 & 9 & 10 \\
Item I & 0 & 1 & 1 & 2 & 2 & 3 & 3 & 3 & 4 & 4 & 4 \\
Item 2 & 0 & 0 & 1 & 1 & 2 & 2 & 2 & 2 & 3 & 3 & 4 \\
Item 3 & 0 & 0 & 1 & 1 & 2 & 2 & 3 & 3 & 3 & 4 & 4 \\
Item 4 & 0 & 0 & 2 & 2 & 3 & 3 & 3 & 3 & 3 & 4 & 4 \\
Item 5 & 0 & I & I & 1 & 2 & 2 & 2 & 3 & 3 & 4 & 4 \\
Item 6 & 0 & 0 & I & 1 & 2 & 2 & 3 & 3 & 3 & 4 & 4 \\
Item 7 & 0 & 0 & 1 & 1 & 1 & 2 & 2 & 2 & 3 & 3 & 3 \\
\hline
\end{tabular}

most likely response for persons in some range of the trait being measured. Based on plots of the CCFs we identified response categories for which this was not the case. As the next step in selecting response categories to collapse, we chose a single pair of adjacent categories, neither of which were ever the most likely response at any level of theta, and collapsed these into a single category. The data were then recalibrated using the PCM, and the process was repeated. For each item, the collapsing of categories continued until every remaining item category was the most probable response for at least some range of theta. In the completed dataset, one item had four response categories, and all other items had five response categories. Table 2 reports how each item was recoded to obtain the final dataset.

For all datasets, fit to the partial credit model was evaluated based on WINSTEPS-generated fit statistics. The InFit Mean Square reflects the degree to which response patterns are similar and is based on the ratio of observed residual variance to expected residual variance..$^{37,38}$ Values outside the range of 0.6 to 1.4 have conventionally been viewed as indicating inadequate model fit. ${ }^{39}$ Person separation and reliability estimates were calculated based on calibrations of each dataset. The person reliability statistic estimates the degree to which the instrument discriminates among peoples' trait levels and the degree to which the order of persons on the trait continuum can be replicated using a different instrument measuring the same construct. ${ }^{40,41}$ Person reliability is approximately equivalent to more traditional reliability estimates such as KR-20 and Cronbach's alpha. Values of the person reliability range from 0 to 1 , with values closer to 1 indicating better discrimination at extreme levels of the trait (eg, low pain and high pain).

To compare the validity of scores obtained based on different numbers of response categories, we conducted nonparametric significance tests using the scores as the predictor variables. We hypothesized that BPI interference scores would be related to self-reported general health, 
average pain in the past seven days, and worst pain in the past seven days. We also compared BPI interference scores obtained from the various datasets with respect to their ability to distinguish among levels of pain interference in the three clinical populations: cancer, MS, and limb amputation (upper or lower).

\section{Results}

\section{Study population}

The population was predominantly female (66\%) and Caucasian (90\%) and relatively evenly split between the two geographic sites (54\% from ENH, Chicago). The predominant diagnosis was cancer (47\%). Among those for whom the stage of cancer was reported $(82 \%)$, there was a relatively even spread among stages (Stage 1: 21\%, Stage 2: 31\%, Stage 3: 24\%, and Stage 4 and higher: 24\%). Thirty-one percent of the population had MS, and eight percent had received an (upper/lower) limb amputation.

\section{Score distribution}

Figure 1 presents the distribution of scores by item and response category. As expected, scores were skewed negatively; that is, more persons endorsed lower response categories indicating lower levels of pain interference.

\section{Data/model fit}

The most misfitting item in every calibration was the item that queried respondents about how much their pain interfered with sleep (BPI-6). Infit values for this item ranged between 1.29 and 1.58 and fell within the preferred 0.6 to 1.4 range for only two datasets: the dataset in which all items were scored with two categories, and the dataset in which all items were scored with three categories (infit values $=1.29$ and 1.38 , respectively).

\section{Person reliability and separation}

\section{Variable Response datasets}

The person reliability estimates for the Variable Response dataset was 0.86 . The person separation estimate for the dataset with variable numbers of response categories was 2.45 .

\section{Uniform Response datasets}

For the Uniform Response datasets, person reliability estimates ranged from 0.38 ( 2 categories) to 0.88 (6 and 7 categories). However, there was little variation in reliability among the datasets scored with 5 to 11 categories ( 0.86 to 0.88 ). The only exceptionally-poor result was for the two-category dataset.
The three- and four-category datasets had values of 0.81 and 0.84 , respectively. Figure 2 portrays these results graphically.

Person separation estimates for the Uniform Response datasets ranged from 0.77 ( 3 categories) to 2.66 (6 and 7 categories). Values dropped off slightly for the datasets with the largest number of categories. For the 10- and 11-category datasets, the values were 2.47 and 2.45 , respectively. The biggest decrement in separation, however, was for the datasets with two and three categories ( 0.77 and 0.79 , respectively). These results are displayed in Figure 3.

\section{Self-reported general health and diagnoses}

Self-reports of general health (collected at University of Washington site) and diagnoses (collected at both sites) were used to evaluate how well the scores from different datasets discriminated among levels of health and among the three clinical populations. University of Washington participants responded to the 36-item Medical Outcomes Study Short-Form Health Survey (SF-36). ${ }^{42}$ One item asked respondents to rate their general health as 'excellent', 'very good', 'good', 'fair', or 'poor'. Respondents were classified into five groups based on their responses to this item. To evaluate pain interference scores with respect to discrimination among levels of self-reported general health, we calculated the nonparametric Kruskal-Wallis H statistic. The probability of the null was taken as an indicator of how well the scores were discriminating. Our assumption was that lower $P$-values would indicate more successful discrimination among groups. A second set of analyses compared pain interference scores from different datasets with respect to their ability to discriminate among the three clinical groups in our study population: MS, amputation, and cancer.

\section{Variable Response datasets}

For the Variable Response dataset, the $P$-value obtained in the comparison of self-reported general health was 0.0004 ; while for the comparison of scores by diagnosis, the value was $5.4 \times 10^{10}$.

\section{Uniform Response datasets}

Figure 4 presents results for the Uniform Response datasets. For all except the two-category dataset, statistical comparisons between pain interference scores and General Health scores resulted in $P$-values less than 0.001. All but the scores from the two-, three-, and four-category 

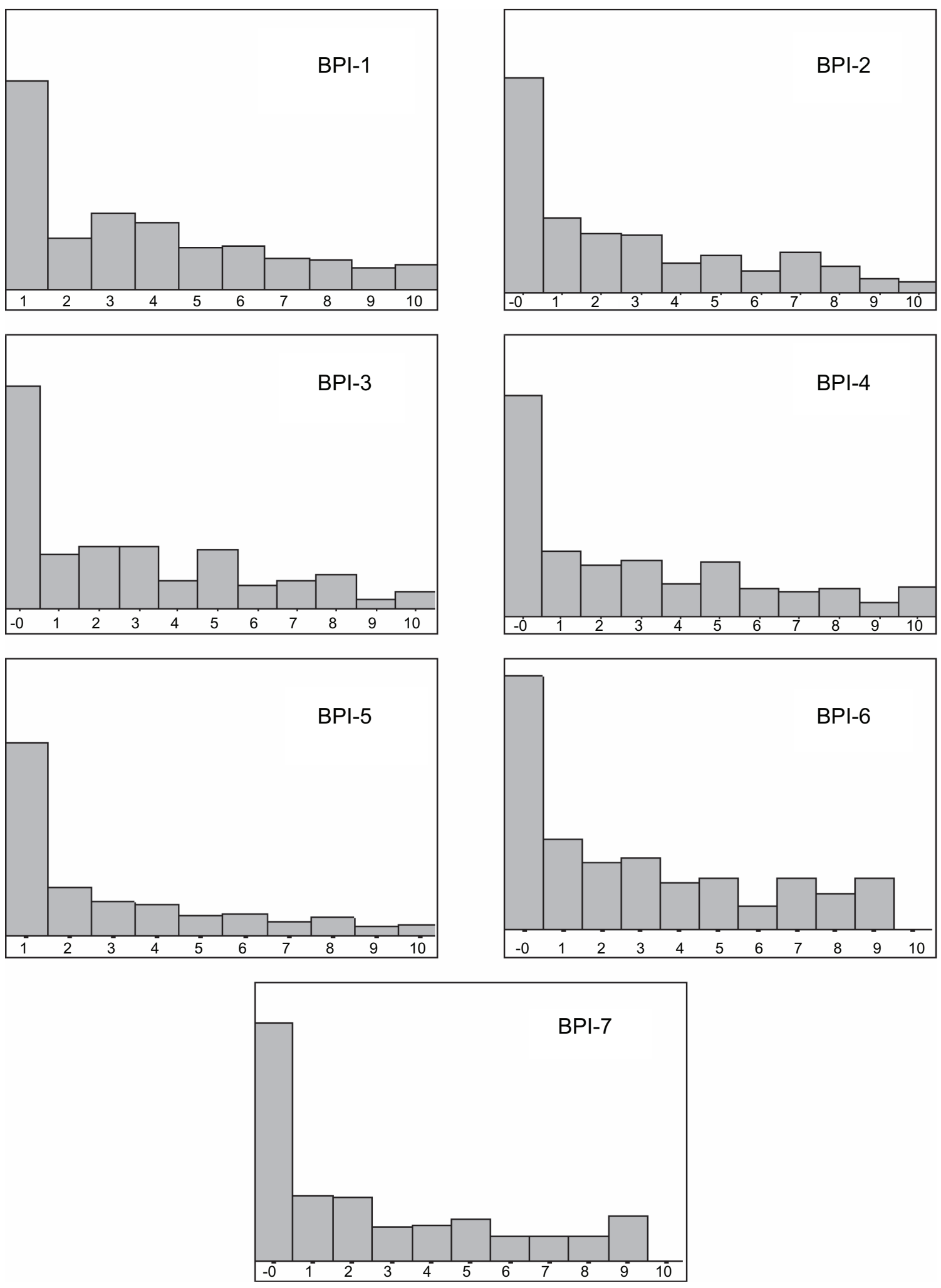

Figure I Distribution of observed item responses to the seven items of the Brief Pain Inventory (BPI) Interference scale.

datasets distinguished diagnostic groups at $P$-values less than 0.002 . The two-, three-, and four-category datasets had $P$-values of $0.25,0.07$, and 0.10 , respectively. In all but one comparison, the amputation group had the highest pain interference scores, followed by MS, and then cancer. In the two-category scoring, the amputation group had the highest pain interference scores, followed by cancer, and then MS. 


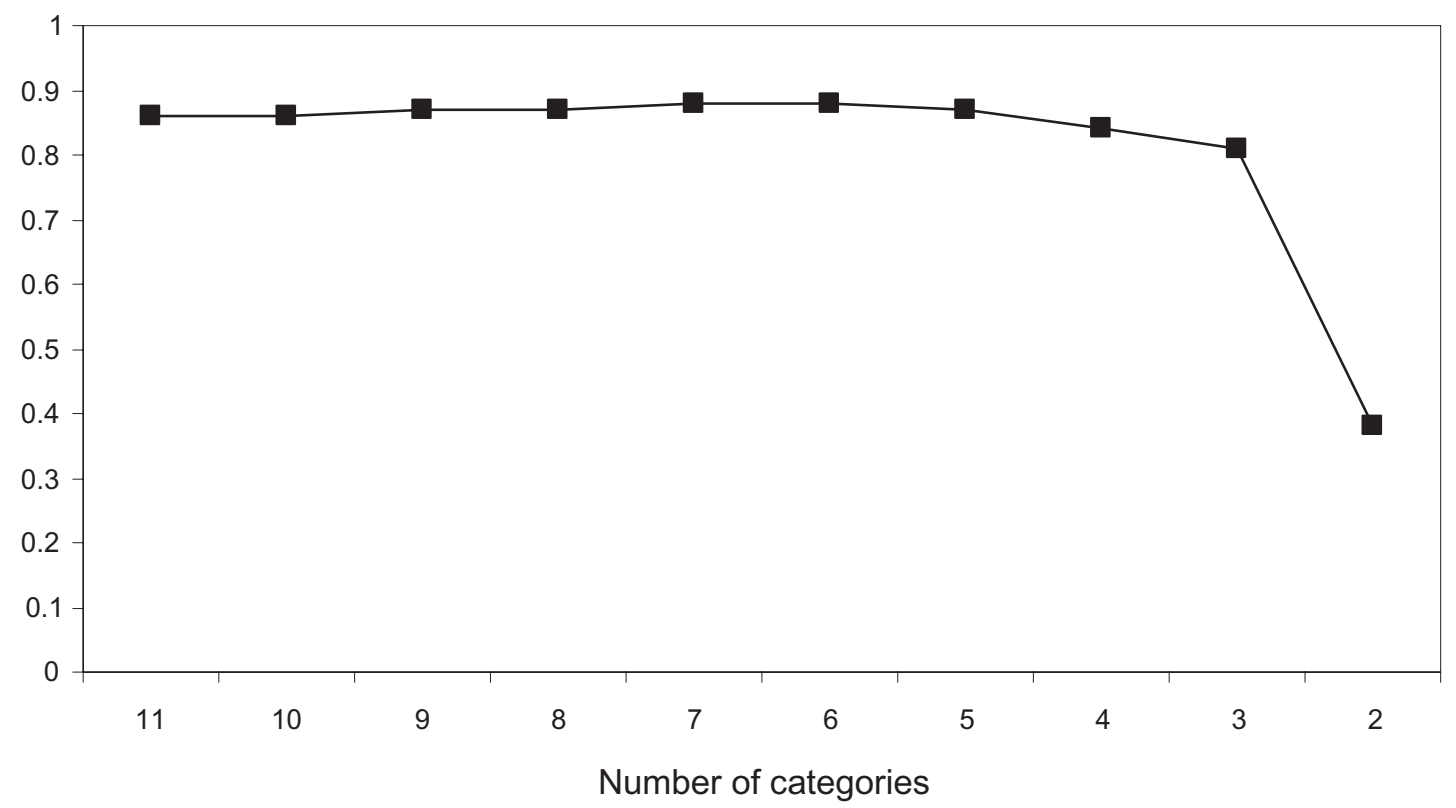

Figure 2 Person reliability estimates by number of response categories estimates by number of response categories.

\section{Self-reported average and worst pain in past seven days}

In addition to the pain interference subscale, the BPI includes items that query participants regarding their 'worst' and 'average' pain during the past seven days. Responses range from $0-10$ with 0 indicating 'no pain,' and 10 indicating 'pain as bad as you can imagine.' Persons were grouped into 11 categories based on their responses to these items. The Kruskal-Wallis H statistic was calculated to evaluate the degree to which pain interference scores from each dataset differentiated among levels of average and worst pain. These results are summarized in Figure 5.

\section{Variable Response datasets}

For the dataset with variable numbers of response categories, $P$-values obtained from the comparisons of pain interference and levels of average and worst pain were $6.8 \times 10^{11}$ and $9.6 \times 10^{12}$, respectively.

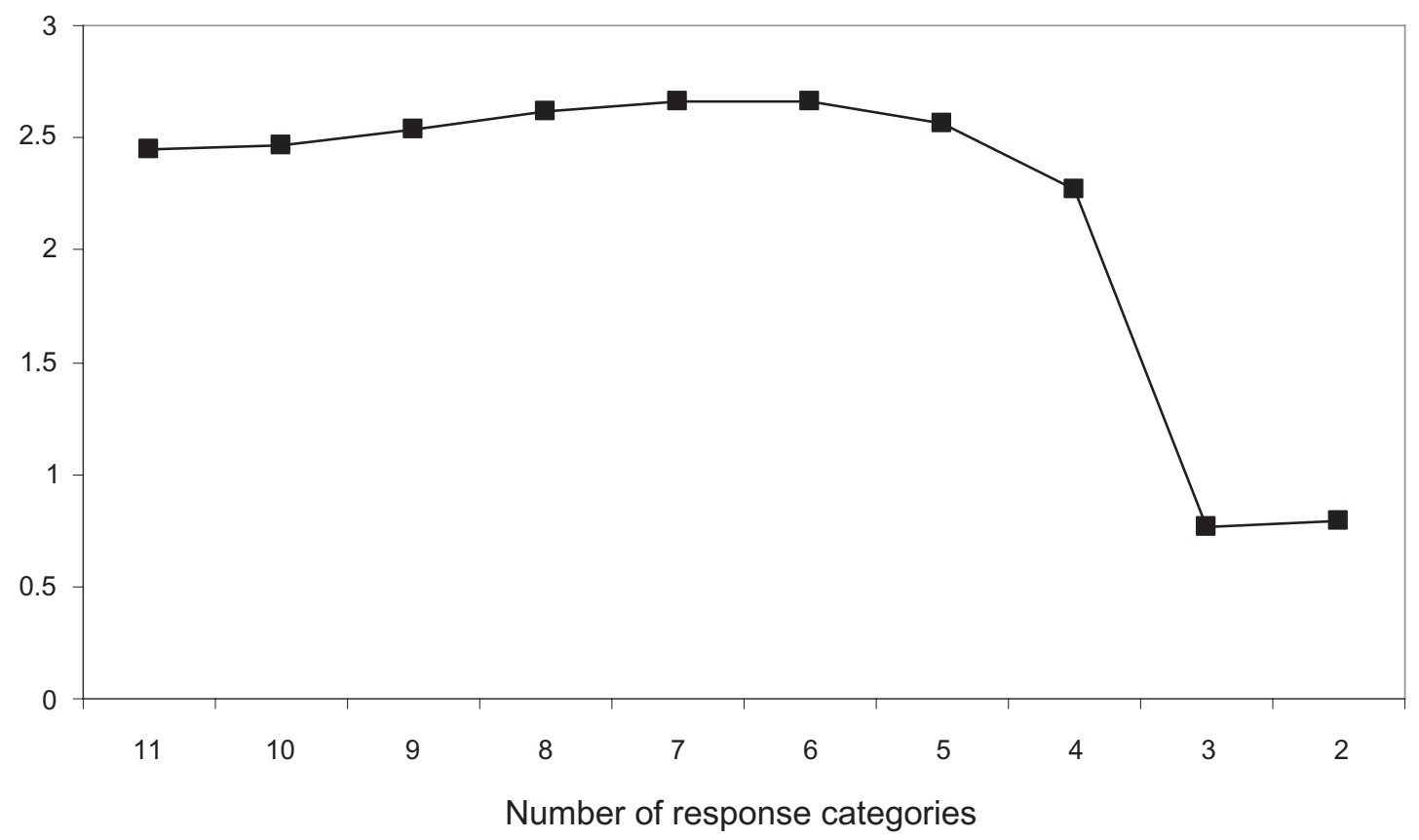

Figure 3 Person separation estimates by number of response categories. 


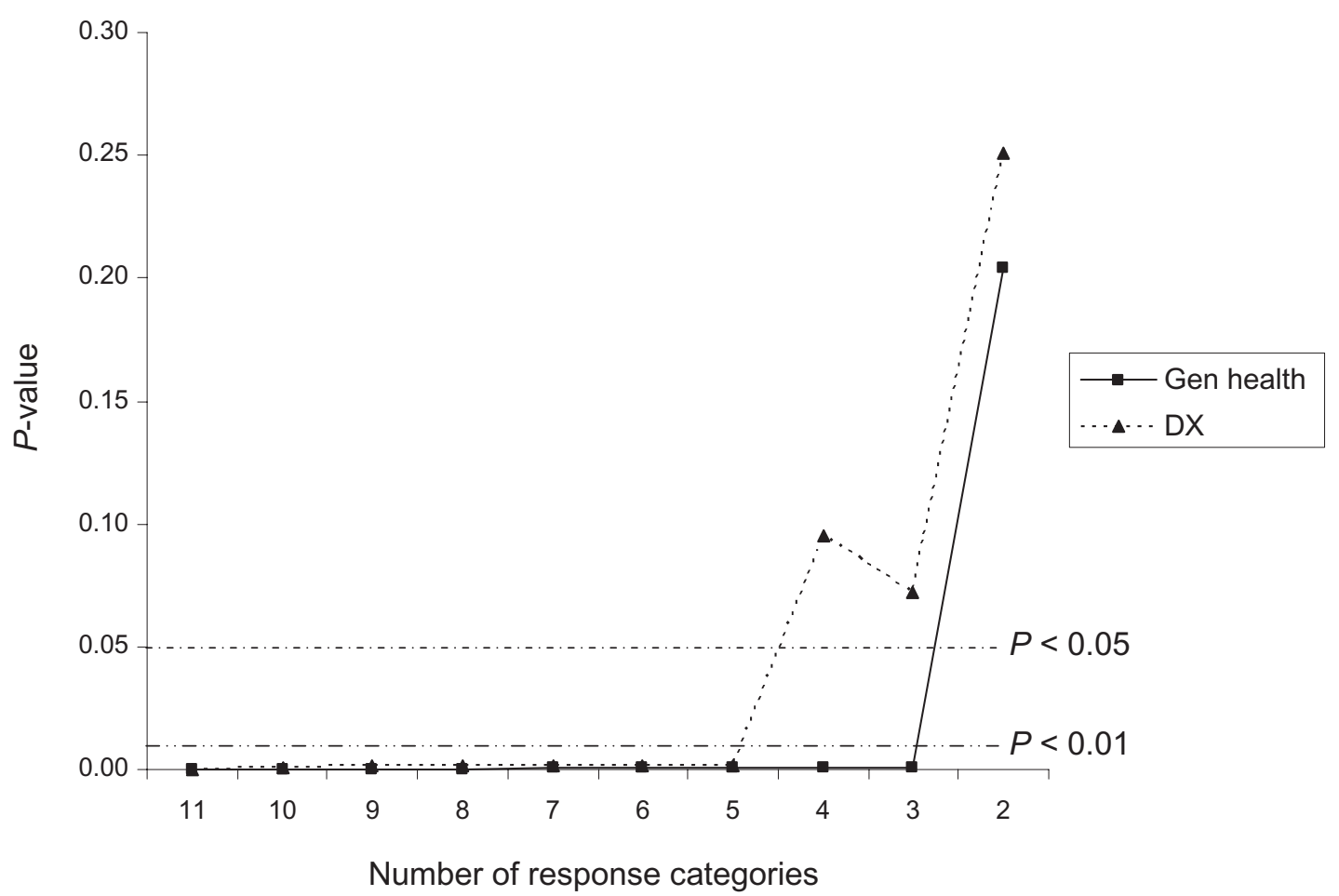

Figure 4 Probability of the null (Kruskall-Wallis) in comparisons of Brief Pain Inventory Interference scores and self-reported general health and diagnosis.

\section{Uniform Response datasets}

Among the Uniform Response datasets, the comparison of groups classified based on responses to the 'worst pain' item yielded $P$-values substantially less than 0.001 for every dataset except the two-category one. For this dataset, the $P$-value for the worst pain comparison was 0.06. In the comparisons based on reported average pain, the value was 0.08 for the two-category dataset and 0.004 for the three-category dataset. All other $P$-values were well below 0.001 .

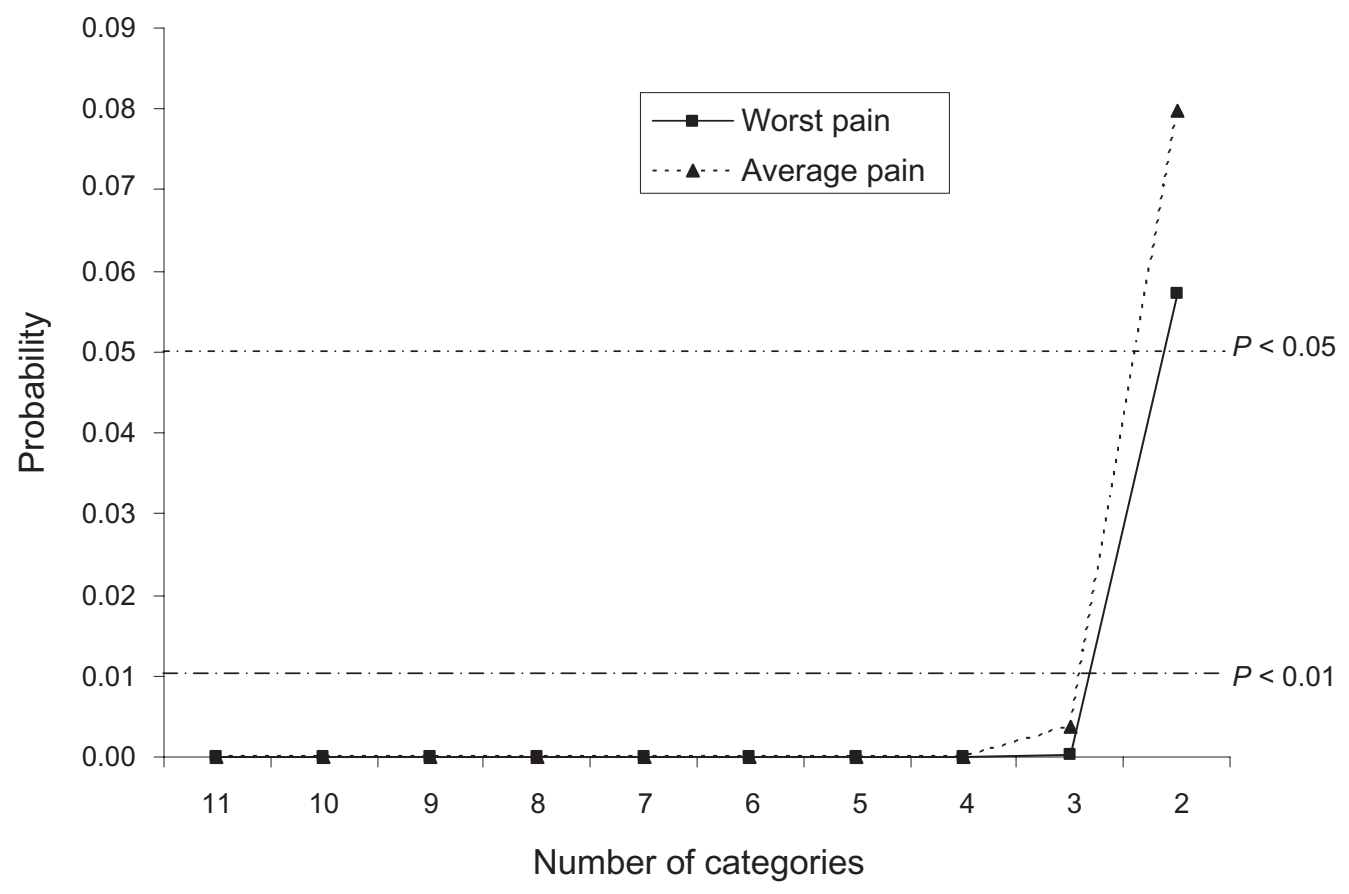

Figure 5 Probability of the null (Kruskall-Wallis) in comparisons of self-reported average and worst pain. 


\section{Conclusions}

In the current study, measuring pain interference scores obtained after collapsing down to as few as five categories was roughly equivalent to or slightly more effective than scoring based on all original 11 categories. The fact that, in all comparisons, scores obtained based on five to seven categories performed at least as well and often slightly better than did scores based on more categories raises the question of what is the optimum number of response categories for self-reporting pain interference. The design for the current study is not adequate for answering this question. Studies need to be conducted that systematically vary the number of responses presented for persons self-reporting pain interference. The resulting scores should be compared with regard to their concurrent validity and success in discriminating among known groups. However, our results do provide preliminary evidence that respondents may not distinguish 11 levels of pain interference, and asking them to attempt to do so may increase measurement error. Presenting a large number of response categories also increases the 'cognitive load' of self-report and may add appreciably to response burden.

Our results highlight the need for investigations regarding pain perception and self-report. Though it is common in pain assessment to present items with eleven categories or more, it is an empirical question whether people are actually able to discriminate this many levels of pain. The items used in the current study were developed to measure pain interference, not a 'simple sensory attribute'. However, the results suggest that self-perceptions of pain interference may be similarly limited in channel capacity. Research regarding persons' cognitive representations and discriminations of pain outcomes would inform efforts to measure it.

\section{Acknowledgments/disclosures}

The authors report no conflicts of interest in this work. This study was supported by grants from the National Institutes of Health: National Institute of Child Health and Human Development (PO1 HD33988); National Cancer Institute (CA60068); and Patient-Reported Outcomes Measurement Information System (PROMIS), a National Institutes of Health (NIH) Roadmap initiative (U01AR52171). Support also was received from the National Institute of Disability and Rehabilitation Research, Department of Education (H133B031129 and H133B031120) and the American Cancer Society (\#RSG-01-221-01-PBP)

\section{References}

1. Chang HM. Cancer pain management. Med Clin North Am. 1999;83(3):711-736, vii.

2. Meuser T, Pietruck C, Radbruch L, Stute P, Lehmann KA, Grond S. Symptoms during cancer pain treatment following WHO-guidelines: a longitudinal follow-up study of symptom prevalence, severity and etiology. Pain. 2001;93(3):247-257.

3. Dworkin RH, Turk DC, Farrar JT, et al. Core outcome measures for chronic pain clinical trials: IMMPACT recommendations. Pain. 2005;113(1-2):9-19.

4. Preston CC, Colman AM. Optimal number of response categories in rating scales: reliability, validity, discriminating power, and respondent preferences. Acta Psychologica. 2000;104:1-15.

5. Weng LJ. Impact of the number of response categories and anchor labels on coefficient alpha and test-retest reliability. Educ Psychol Meas. 2004;64(6):956-972.

6. Symonds PM. On the loss of reliability in ratings due to coarseness of the scale. $J$ Exp Psychol. 1924;7:456-461.

7. Bending AW. The reliability of self-ratings as a function of the amount of verbal anchoring and the number of categories on the scale. $J \mathrm{Appl}$ Psychol. 1953;37:38-41.

8. Bending AW. Reliability and the number of rating scale categories. J Appl Psychol. 1954;38:38-40.

9. Cicchetti DV, Showalter D, Tyrer PJ. The effect of number of rating scale categories on level of inter-rater reliability: a Monte-Carlo investigation. Appl Psychol Meas. 1985;9:31-36.

10. Finn RH. Effects of some variations in rating scale characteristics on the means and reliabilities of ratings. Educ Psychol Meas. 1972;34:885-892.

11. Nunnally J. Psychometric Theory. New York, NY: McGraw-Hill; 1967.

12. Ramsay J. The effect of number of categories in rating scales on precision of estimation of scale values. Psychometrika. 1973;38:513-533.

13. Chang L. A psychometric evalutaiton of four-point and six-point Likert-type scales in relation to reliability and validity. Appl Psychol Meas. 1994;18:205-215.

14. Jenkins J, FD, Taber T. A Monte-Carlo study of factors affecting three indices of composite scale reliability. J Appl Psychol. 1977;62:392-398

15. Lissitz R, Green S. Effect of the number of scale points on reliability: a Monte-Carlo approach. J Appl Psychol. 1975;60:10-13.

16. McKelvie S. Graphic rating scales: How many categories? Br J Clin Psychol. 1978;69:185-202.

17. Miller GA. The magical number seven, plus or minus two: some limits on our capacity for processing information. Psychol Rev. 1956;63:81-97.

18. Cleeland CS. Measurement of pain by subjective report. In: Chapman CR, editor. Advances in Pain Research and Management. New York, NY: Raven Press; 1989;12:391-403.

19. Yun YH, Mendoza TR, Heo DS, et al. Development of a cancer pain assessment tool in Korea: a validation study of a Korean version of the brief pain inventory. Oncology. 2004;66(6):439-444.

20. Badia X, Muriel C, Gracia A, et al. Validation of the Spanish version of the Brief Pain Inventory in patients with oncological pain. Med Clin (Barc). 2003;120(2):52-59.

21. Klepstad P, Loge JH, Borchgrevink PC, Mendoza TR, Cleeland CS, Kaasa S. The Norwegian brief pain inventory questionnaire: translation and validation in cancer pain patients. J Pain Symptom Manage. 2002;24(5):517-525.

22. Tan G, Jensen MP, Thornby JI, Shanti BF. Validation of the Brief Pain Inventory for chronic nonmalignant pain. J Pain. 2004;5(2): 133-137.

23. Raichle KA, Osborne TL, Jensen MP, Cardenas D. The reliability and validity of pain interference measures in persons with spinal cord injury. J Pain. 2006;7(3):179-186. 
24. Tittle MB, McMillan SC, Hagan S. Validating the Brief Pain Inventory for use with surgical patients with cancer. Oncol Nurs Forum. 2003;30(2):325-330.

25. Tyler EJ, Jensen MP, Engel JM, Schwartz L. The reliability and validity of pain interference measures in persons with cerebral palsy. Arch Phys Med Rehabil. 2002;83(2):236-239.

26. Mendoza TR, Chen C, Brugger A, et al. The utility and validity of the modified Brief Pain Inventory in a multiple-dose postoperative analgesic trial. Clin J Pain. 2004;20(5):357-362.

27. Keller S, Bann CM, Dodd SL, Schein J, Mendoza TR, Cleeland CS Validity of the Brief Pain Inventory for use in documenting the outcomes of patients with noncancer pain. Clin J Pain. 2004;20(5):309-318.

28. Williams VS, Smith MY, Fehnel SE. The validity and utility of the BPI interference measures for evaluating the impact of osteoarthritic pain. J Pain Symptom Manage. 2006;31(1):48-57.

29. Ger LP, Ho ST, Sun WZ, Wang MS, Cleeland CS. Validation of the Brief Pain Inventory in a Taiwanese population. J Pain Symptom Manage. 1999;18(5):316-322.

30. Mystakidou K, Mendoza T, Tsilika E, et al. Greek brief pain inventory: validation and utility in cancer pain. Oncology. 2001;60(1):35-42.

31. Zelman DC, Gore M, Dukes E, Tai KS, Brandenburg N. Validation of a modified version of the Brief Pain Inventory for painful diabetic peripheral neuropathy. J Pain Symptom Manage. 2005;29(4):401-410.

32. Radbruch L, Loick G, Kiencke P, et al. Validation of the German version of the Brief Pain Inventory. J Pain Symptom Manage. 1999;18(3):180-187.
33. WINSTEPS: Rasch-model computer program [computer program]. Version 3.3. Chicago, IL: MESA Press; 2001.

34. Masters GN. A Rasch model for partial credit scoring. Psychometrika. 1982;47:149-174.

35. Embretson SE, Reise SP. Item Response Theory for Psychologists. Mahway, NJ: Lawrence Erlbaum Associates, Publishers; 2000.

36. Hambleton R, Swaminathan H, Rogers HJ. Fundamentals of Item Response Theory. Newbury Park, CA: Sage Publishing, Inc.; 1991.

37. Wright BD. Solving measuring problems with the RASCH Model. Journal of Educational Measurement. 1977;14:97-116.

38. Smith RM. The distributional properties of Rasch item fit statistics. Journal of Educational and Psychological Measurement. 1991;48:657-667.

39. Wright BD. Reasonable mean-square fit. Rasch Measurement Transactions. 1994;8:1.

40. Bond TG, Fox CM. Applying the Rasch Model: Fundamental measurement in the human sciences. Mahwah, NJ: Lawrence Erlbaum Assoc; 2001.

41. Stone M, Yumoto F. The effect of sample size for estimating Rasch/IRT parameters with dichotomous items. J Appl Meas. 2004;5:48-61.

42. Ware JE Jr, Sherbourne CD. The MOS 36-item short-form health survey (SF-36). I. Conceptual framework and item selection. Med Care. $1992 ; 30(6): 473$ 
Appendix

Brief Pain Inventory Interference subscale items

During the past seven days, how much has pain interfered with your:

General activity

Mood

Walking ability
Normal work (includes both work outside the home and housework)

Relations with other people

Sleep

Enjoyment of life

Response scale: $0-10$, where $0=$ 'Does not interfere' and $10=$ 'Interferes completely'.

\section{Publish your work in this journal}

Patient Related Outcome Measures is an international, peer-reviewed, open access journal focusing on treatment outcomes specifically relevant to patients. All aspects of patient care are addressed within the journal and practitioners from all disciplines are invited to submit their work as well as healthcare researchers and patient support groups. Areas covered will include: Quality of life scores; Patient satisfaction audits; Treatment outcomes that focus on the patient; Research into improving patient outcomes; Hypotheses of interventions to improve outcomes; Short communications that illustrate improved outcomes; Case reports or series that show an improved patient experience; Patient journey descriptions or research. 